\title{
Sánchez Juárez, Gladys Karina (2015). Los pequeños cafeticultores de Chiapas. ORgANIZACIÓN Y RESISTENCIA FRENTE AL MERCADO. MÉXICO: CESMECA-UNICACH
}

\author{
Dolores Camacho-Velázquez
}

E ste libro es una versión de la tesis titulada: Sociedad, mercado y Estado. Las organizaciones de cafeticultores de comercio justo y orgánico en Chiapas, que la autora presentó en su momento para obtener el grado de doctora en Ciencias Sociales y Humanísticas. La investigación pretende explicar y caracterizar la relación Estado/mercado/sociedad, por medio del análisis de las organizaciones de pequeños productores de café en Chiapas. Para ello, eligió la Coordinadora de Pequeños Productores de Café de Chiapas (Coopcafe), y específicamente cuatro organizaciones que forman parte de ella: Tzeltal-Tzotzil, Cholom Bolá, Comon Yaj Nop Tic y Unión Ramal Santa Cruz. La elección se justifica en que, por encontrarse estas organizaciones en territorios diferentes, son representativas de la diversidad territorial y, por lo tanto, cultural del estado. Por otro lado, como afirma la autora, son representativas de la Coordinadora.

El texto se compone de cinco capítulos. En el primero de ellos, titulado "La sociedad rural y el Estado mexicano", hace un recorrido histórico por la conformación del Estado nacional mexicano en un contexto global, identificando las diversas fases del capitalismo internacional. En el trayecto va relacionando estas fases con el agro, es decir, señala las tendencias de las políticas nacionales y globales y su aplicación en el campo. Identifica las principales características del Estado fordista keynesiano, que en México se identifica con la aplicación del modelo de sustitución de importaciones. Bajo la aplicación de este modelo, se privilegia el apoyo al campo como una forma de impulsar y sostener el desarrollo de la industria.

En esta época, que va de los años cuarenta hasta finales de los sesenta o principios de los setenta, el campo mexicano vivió un buen momento, puesto que el corporativismo funcionó para integrar a los campesinos al Estado nacional, y estos vivieron durante ese proceso una etapa de bonanza. En particular en Chiapas, los ejidos y comunidades que ya habían sido beneficiados con el reparto agrario fueron organizaciones territoriales y políticas exitosas hasta principios de los noventa, aunque obviamente había otras que en esos tiempos estaban luchando por el acceso a la tierra y por mejores condiciones de vida en las fincas donde aún eran mozos.

En el caso de los cafeticultores, la autora identifica el año 1989 como un momento en que puede identificarse un antes y un después en esta historia, porque en ese año el precio del café cayó de manera drástica y el Estado se retiró del proceso de regulación del mercado. De ahí derivó la modificación al artículo 27 constitucional, y finalmente en 1994 el campo se hundió con la entrada en vigor del Tratado de Libre Comercio. Los apoyos fueron

Dolores Camacho Velázquez, Centro de Estudios Multidisciplinarios sobre Chiapas y la Frontera Sur, de la Universidad Nacional Autónoma de México. Correo electrónico: doloresc@unam.mx.

Recibida: 23 de septiembre de 2015. 
retirados de manera brusca y se liberaron los precios de los insumos principales, a lo que se llamó el fin del pacto social y el principio real del neoliberalismo.

En este capítulo, la autora explica cómo se redujo el gasto dirigido a la producción, y el presupuesto para el combate a la pobreza se extendió a lugares donde se volvió necesario por la lógica razón de que los campesinos, al dejar de recibir el subsidio a la producción, se convirtieron en "pobres", sobrevivientes gracias a la política social. Fueron tiempos en que se afirmaba que los campesinos desaparecerían; sin embargo, continuaron, tuvieron que amoldarse a esas nuevas normas y encontrar espacios de participación que les permitieran seguir existiendo y reproduciendo la unidad doméstica. Este planteamiento es el marco para entender la historia de los pequeños cafetaleros chiapanecos. La autora lo observa desde el enfoque histórico estructural, lo cual parece un acierto, dado que esa perspectiva permite ubicar a los campesinos en la dimensión latinoamericana y en el contexto global del capitalismo.

En el segundo capítulo, "Los campesinos mexicanos en el mercado internacional del café”, se analiza el cambio que hubo a partir de la aplicación de las políticas neoliberales. La autora describe cómo tuvieron que adaptarse los campesinos a los nuevos procesos. En 1989, el primer gran golpe fue la desaparición del Instituto Mexicano del Café, que era el organismo encargado de regular la producción y el precio. Fue una auténtica sacudida, no porque éste funcionara y cumpliera con su papel de forma ejemplar, sino porque, al liberar el precio y el proceso regulatorio, los pequeños productores se vieron expuestos a competir con los grandes productores. Por ser el café un producto completamente comercial, los cafetaleros tuvieron que adaptarse rápidamente a esa nueva condición. El Estado, dice Sánchez, creó las condiciones para que estos pequeños productores constituyeran organizaciones que les permitieran convertirse en intermediarias directas de los campesinos ante el Estado, sustituyendo así a las grandes corporaciones, como la Confederación Nacional Campesina (CNC), que dejaron de cumplir ese papel. Los cafetaleros se involucraron y jugaron ese nuevo rol; sin embargo, la autora argumenta que no lo hicieron como actores pasivos, sino porque ellos mismos consideraron la utilidad de esa estrategia para continuar funcionando y existiendo. Seguirían gozando de cierta autonomía en el proceso productivo y en su relación con el mercado, aunque fue relativa debido a que el Estado y el mercado son realmente los que imponen las condiciones por medio de los apoyos a organizaciones y a través del precio.

En esa circunstancia, se organizaron para enfrentar al mercado y se involucraron en el comercio justo y la producción orgánica, lo cual parece lógico dadas las características de este tipo de mercado. La autora señala que "en la dinámica del comercio justo hay un intento por desmercantilizar las relaciones sociales", aunque, según lo expuesto por ella misma, lo que los pequeños cafetaleros analizados pretendían era acceder a un sistema comercial que les permitiera obtener mejores ingresos por vía del precio y que se revalorara el trabajo campesino. Es decir, desde estas experiencias no fue precisamente el comercio justo lo que funcionó en los lugares de origen, pero significó una opción que debía aprovecharse.

El tercer capítulo, “iIntegración o subordinación de los campesinos organizados?", plantea la historia del movimiento campesino y el marco en el cual surgen sus organizaciones, específicamente las cafetaleras. La autora explica las formas en que se constituyeron, identifica las razones y las estrategias de acción, y reflexiona sobre la forma en que reivindicaron su autonomía. Se refiere a una autonomía productiva y del sistema de partidos, lo que significó una manera de diferenciarse de su participación y corporativismo en la CNC. Sin embargo, también reconoce y describe cómo poco a poco estas organizaciones fueron redefiniendo sus relaciones con el Estado y se sometieron a las pautas institucionales debido a que las consideraron como una opción para sostenerse, al grado de terminar en un nuevo proceso de corporativismo. La autora justifica este nuevo corporativismo afirmando que era la única manera de acceder a los recursos del Estado y de crear estrategias de participación en el plano internacional; sin embargo, reflexiona que esas opciones están llegando 
a su límite debido a que es difícil competir con grandes productores y corporaciones comercializadoras.

En el cuarto capítulo, "Las organizaciones de café: estrategia política y económica de los cafeticultores minifundistas", la autora expone los datos obtenidos en el trabajo de campo y reflexiona en función de los intereses de su investigación; de hecho, una de las aportaciones más importantes de este libro es la cantidad de información de primera mano que maneja y que puede ser útil para hacer otras reflexiones, e incluso interpretaciones. En este capítulo describe el origen y funcionamiento de las organizaciones analizadas y las agrupa por sus características culturales. Por un lado, dos organizaciones, la Tzeltal-Tzotzil en Pantelhó y Cholom Bola en Tila, comparten una misma historia de explotación y desposesión, ambas están constituidas por campesinos que, por su origen étnico, expresan una fuerte cohesión social y tienen una extensión de tierra que no rebasa las dos hectáreas.

Otras dos organizaciones, Comon Yaj Nop Tic y Unión Ramal Santa Cruz, se ubican en La Concordia, en la región Frailesca. Los campesinos que las integran tienen una diversificación mayor, cuentan con más tierra y se asientan en territorios de verdadera competencia y productividad.

Las historias y el funcionamiento de estas organizaciones dejan ver la diversidad de pequeños productores que las componen, algunos más arraigados a la tierra y a la colectividad, como es el caso de las poblaciones indígenas, y los otros, más aferrados a actividades económicas variadas, como el caso de las organizaciones de la Frailesca; sin embargo, todos tienen en común que defienden su forma de vida campesina.

En el quinto capítulo, "La racionalidad campesina en torno a un producto global", la autora refuerza su hipótesis de que los pequeños productores de café siguen siendo campesinos a pesar de que funcionan en gran medida por la dinámica del mercado y por el funcionamiento del Estado. Con datos y entrevistas, demuestra que la forma de producción sigue siendo en gran medida familiar, aunque se utiliza mano de obra pagada, que se contrata en momentos en que la familia ya no es capaz de hacer todo por sí misma. Normalmente los empleados son personas de la misma comunidad que en algunos momentos son dueños de la tierra y en otros trabajadores de otros compañeros; sólo en algunos casos ubicados en las organizaciones de La Concordia, estos trabajadores provienen de Guatemala.

El estudio ofrece datos que demuestran cómo la división de dos territorios, con dos organizaciones cada uno, tiene fundamento no sólo histórico-cultural, sino en sus formas de relacionarse con el mercado; los cafeticultores de las organizaciones ubicadas en zonas indígenas de Pantelhó y Tila, además de lo ya mencionado, comparten una forma de relacionarse entre ellos y la tierra: también participa toda la familia en el proceso de trabajo, tienen una diversificación productiva mayor y son en gran medida autosuficientes en su alimentación, es decir, no dependen completamente del mercado para vivir. Si el precio del café cae - lo cual suele sucederpueden vivir con lo que producen en el traspatio y con el maíz. En tal sentido, la autora ubica a estos productores como campesinos en toda la extensión de la palabra, ya que la diversificación productiva y el acceso al mercado a través de la venta de un producto comercial como el café son estrategias para mantener su condición de campesinos.

En el caso de organizaciones de La Concordia, Sánchez determina que se trata de poblaciones descendientes de indígenas que emigraron a esa zona para trabajar en las fincas, se quedaron allá y fueron luchando por la tierra hasta convertirse en propietarios por medio de la dotación de tierras o, en algunos casos, de la compra; sin embargo, reproducen la cultura campesina de esa región que es más especializada y menos diversificada que la de los territorios indígenas. Estos campesinos tienen más tierra en posesión y más cantidad dedicada al cultivo de café en comparación con el primer grupo. Ello significa que, cuando la producción del café es buena y el precio también, los cafetaleros pueden obtener ingresos muy importantes, lo que les permite comprar en el mercado todo lo necesario para sobrevivir y hasta para satisfacer las necesidades de educación profesional de sus hijos mayores; sin embargo, son más vulnerables porque, ante problemas 
en la producción o bajas en el precio, no tienen opciones dado que no producen sus propios alimentos - aunque la autora menciona algunas excepciones-. Estos campesinos son sujetos de crédito y requieren de otros tipos de incentivos para su producción, así como de los apoyos derivados de la política social del gobierno y de otros ingresos para solventar sus gastos. En este grupo sólo los jefes de familia o los hijos en edad de trabajar que no salieron de la casa son quienes participan en la producción de café. Las mujeres hacen el trabajo de la casa y normalmente contratan a gente que apoye en las labores de cultivo y producción del grano, por lo que aparentemente estos productores no son propiamente campesinos. De hecho, la autora observa que: "en el caso de este tipo de cafeticultores se observa la profundización de su 'agriculturación' pues, en efecto, la participación de la economía campesina en el sistema de economía de mercado también supone la 'agriculturación' del campesino" (182). Por sus formas de relacionarse con la tierra y sus estrategias de sobrevivencia, están en posición de afirmar que, aun teniendo una relación más directa con el mercado a través del crédito y la venta de su producción, siguen siendo campesinos porque la acumulación y la renta no existe para ellos; sus estrategias son de sobrevivencia y de reproducción de la unidad domestica, aunque los hijos pueden ir a la universidad y dedicarse a otras actividades no relacionadas con el campo, es decir, son campesinos, pero con otras características.

La conclusión de la autora es que la cultura campesina se manifiesta en todos los casos y su forma de relacionarse con el mercado es de supervivencia y no de acumulación. También observa que el grado de autonomía que los campesinos tienen es restringida porque la libertad para la toma de decisiones está limitada por el mercado y por el Estado.

La autora hace un agudo análisis de las condiciones actuales que enfrentan los productores de café, en el marco del proceso de desarticulación estatal y de descomposición social por el que atraviesa el Estado mexicano, como consecuencia de que las políticas neoliberales profundizan las desigualdades y someten a los campesinos a una vida de supervivencia. Lo que el estudio demuestra es que los campesinos siempre encuentran esos espacios que dejan el mercado y el Estado para seguir subsistiendo. Los resultados de esta investigación se enmarcan también entre los estudios rurales que ofrecen experiencias de vida campesina y que dan argumentos para afirmar que el sistema de vida campesino es tan fuerte, resistente y autónomo que deben reconsiderarse las políticas públicas que han intentado que desaparecieran para integrarlos a un sistema de vida que ellos desconocen. En este caso, la relación con el mercado justo y la producción orgánica es una de las muchas estrategias que han seguido en su vía para resistir, y no precisamente como "la opción". Es importante reconocerlo ahora que el mercado justo y sus reglamentaciones son más parecidos a cualquier otro sistema de mercado, y que en muchos casos no valoran ni reconocen la cultura campesina particular que se desarrolla en estas organizaciones.

Los procesos organizativos analizados por la autora fundamentan las ideas de que en un sistema económico que privilegia al capital y que los excluye desde su condición de pequeños productores, los campesinos sólo podrán sostenerse si se mantienen organizados, asumiendo la organización como una estrategia de resistencia y para mantenerse en el mercado. Pero, además de cuestionar la dignidad de verse reducidos a esa condición de supervivencia, la autora pone en entredicho ciertas prácticas de institucionalización $\mathrm{y}$ de nuevas formas de corporativismo en algunas organizaciones, lo cual es un punto de análisis y de reflexión importante en la mayoría de los procesos organizativos.

Me parece que la crítica a estos procesos no es algo que pueda resolverse tan fácilmente, pues las prácticas involucradas son producto de la condición histórica de estos sujetos sociales, expresión del poder de la cultura política que despliega el Estado. Recordemos que las principales prácticas de las organizaciones sociales mexicanas, y en gran medida latinoamericanas, está basada en el corporativismo y el clientelismo, y, si bien es cierto que el partido único perdió poder en algún momento, eso no significó cambios en esa cultura, porque los resultados demuestran que en algunos casos 
se está reforzando. La obtención de recursos y apoyos del Estado sigue siendo a través de esas prácticas, aunque ahora son muchos los partidos políticos que entran en escena.

En ese sentido, los campesinos tienen que romper con esa cultura política para enfrentarse a lo que viene y constituirse en sujetos de apoyo por el hecho de ser productivos y no por las negociaciones políticas. Es decir, convertirse en sujetos autónomos en toda la extensión del concepto, lo que significa ser capaces de reflexionar sobre su acción y su futuro y no aceptar pasivamente las estrategias impuestas desde las instituciones gubernamentales.

A pesar de que algunas investigaciones afirmaban que los campesinos desaparecerían y que se han aplicado públicas con la finalidad de convertir a los campesinos en pequeños productores individualizados, el sistema de vida campesino ha sido la única forma por la que estos productores han resistido durante tantos años, y podrán continuar generando formas propias de subsistir, con lo que evitarán agrandar los cinturones de miseria en las ciudades. Su función también es social puesto que, si las políticas públicas fomentan el sistema de producción campesina, es posible abonar a la soberanía alimentaria, causa que al parecer ya no importa a ningún político o creador de políticas públicas. El libro analizado nos sirve para fundamentar esta posición y para recordarnos que los pequeños productores de café están resistiendo y sosteniéndose, al igual que tantos otros, en el campo chiapaneco y en el mexicano en general. 